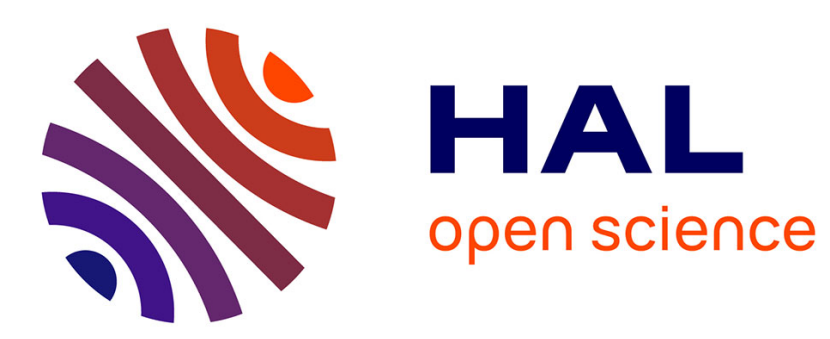

\title{
Measure of Temporal Variation of P-Band Radar Cross Section and Temporal Coherence of a Temperate Tree.
}

Clément Albinet, Pierre Borderies, Nicolas Floury, Eric Pottier

\section{To cite this version:}

Clément Albinet, Pierre Borderies, Nicolas Floury, Eric Pottier. Measure of Temporal Variation of P-Band Radar Cross Section and Temporal Coherence of a Temperate Tree.. IEEE Transactions on Geoscience and Remote Sensing, 2016, 54 (11), p. 6255-6264. 10.1109/TGRS.2016.2565384 . hal01414522

\section{HAL Id: hal-01414522 \\ https://hal.science/hal-01414522}

Submitted on 26 May 2021

HAL is a multi-disciplinary open access archive for the deposit and dissemination of scientific research documents, whether they are published or not. The documents may come from teaching and research institutions in France or abroad, or from public or private research centers.
L'archive ouverte pluridisciplinaire HAL, est destinée au dépôt et à la diffusion de documents scientifiques de niveau recherche, publiés ou non, émanant des établissements d'enseignement et de recherche français ou étrangers, des laboratoires publics ou privés. 


\title{
Measure of Temporal Variation of P-Band Radar Cross Section and Temporal Coherence of a Temperate Tree
}

\author{
Clément Albinet, Pierre Borderies, Nicolas Floury, and Eric Pottier, Fellow, IEEE
}

\begin{abstract}
This paper deals with a ground experiment based on a P-band scatterometer that measured the evolution of the radar cross section (RCS) and coherence of a temperate tree in HV polarization, during four periods spread over nine months, over a cedar tree. Watering of the tree has limited influence in time on the RCS, i.e., limited to around $30 \mathrm{~min}$, but impacts the coherence over a longer period of time. Analysis of the series shows that according to the season considered, clear daily cycles from 1 to $2 \mathrm{~dB}$ may appear on the coherence only (autumn) or on both coherence and RCS (spring), whereas in winter, they are absent on both results. It was analyzed in the literature that the variations in RCS are strongly correlated to the variations in the dielectric constant in trunks and branches. In addition, it was shown that the HV RCS presents seasonal trends with a yearly cycle of roughly $3 \mathrm{~dB}$ following similar trends reported for trunk moisture content time series.
\end{abstract}

Index Terms-Radar, radar cross section (RCS), remote sensing, scatterometer, temperate forests, temporal coherence.

\section{INTRODUCTION}

$\mathbf{I}$ $\mathrm{T}$ IS of great interest to determine the global quantity of biomass on Earth. For this purpose, synthetic aperture radar (SAR) images at the P-band (i.e., around $430 \mathrm{MHz}$ ) look promising in the performance of biomass retrieval over forests. Accurately measuring tropical forest biomass and its temporal change are the main objectives of the BIOMASS mission [1].

At the moment, two main strategies are advanced. On one hand, the "P-HV" method [2] is based on the fact that for most trees, the radar cross section (RCS) for polarization HV is monotonically increasing with biomass. However, previous studies [3] have shown on the base of simulations that other parameters such as branch moisture may also play a significant role in radar backscattering. This then looks worth verifying ex-

C. Albinet and P. Borderies are with the Office National d'Études et de Recherches Aérospatiales (ONERA), 31055 Toulouse Cedex 4, France (e-mail: Clement.Albinet@onera.fr; Pierre.Borderies@onera.fr).

N. Floury is with the European Space Research and Technology Centre, European Space Agency (ESA-ESTEC), 2200 Noordwijk, The Netherlands.

E. Pottier is with the Institut d'Electronique et Télécommunication de Rennes (IETR), 35042 Rennes, France.

Color versions of one or more of the figures in this paper are available online at http://ieeexplore.ieee.org. perimentally by studying variations with time at various scales (intraday, daily, monthly, etc.). On the other hand, the method based on POLINSAR rests on the assumption that biomass is generally proportional to height, and the latter may be derived from knowledge of complex interferometric coherences in $\mathrm{HH}$, HV, and VV [4], [5]. When using repeat-pass interferometry, temporal decorrelation may play a parasitic role in this strategy and, therefore, needs to be evaluated as well.

To precisely study these effects, a proximity experiment is the most convenient as the scrutinized scene may be well characterized. The measurements may be repeated as frequently as wished, and hopefully, they may be linked to some physical characteristics such as air pressure or wind velocity, which may be synchronously measured as well. Two proximity experiments were proposed: one over a tropical forest in French Guyana [6] and another over a cedar tree in Toulouse [7]. This paper deals with the results obtained over nine months with this second experiment. It consists in the observation of one tree with an emitter-receiver located on the roof of the Office National d'Etudes et de Recherches Aérospatiales (ONERA) laboratory. This emitter-receiver is remotely controlled via Ethernet, and then, a fine temporal survey of the intensity and the coherence scattered by a tree may be performed. Its goal is to evaluate and interpret the possible variations of RCS and coherence.

To begin with, the experiment is introduced: The site is displayed, the hardware and the measurement procedure are described, and the parameters considered for the measurements are given. This is done in Section II. Then, in Section III, the system validation is presented. Finally, in Section IV, the results obtained in terms of time series as well as artificial changes in the scene are presented, and a synthesis and an analysis of the obtained results are performed.

\section{Description of the P-Band Scatterometer}

The experiment is based on standard reflectometry from a roof overlooking a tree. The power budget at the distances involved is highly above noise. However, some difficulties arise due to the configuration of the possible sites. The two main difficulties are the insufficient number of looks of the observed scene to correctly evaluate the tree's RCS and coherence and the possible other unwanted contributions of the environment (roof, wall, gate, etc.), which could interfere with tree backscattering. These unwanted contributions are only separated from the tree contribution by a range separation (with time domain) and by 

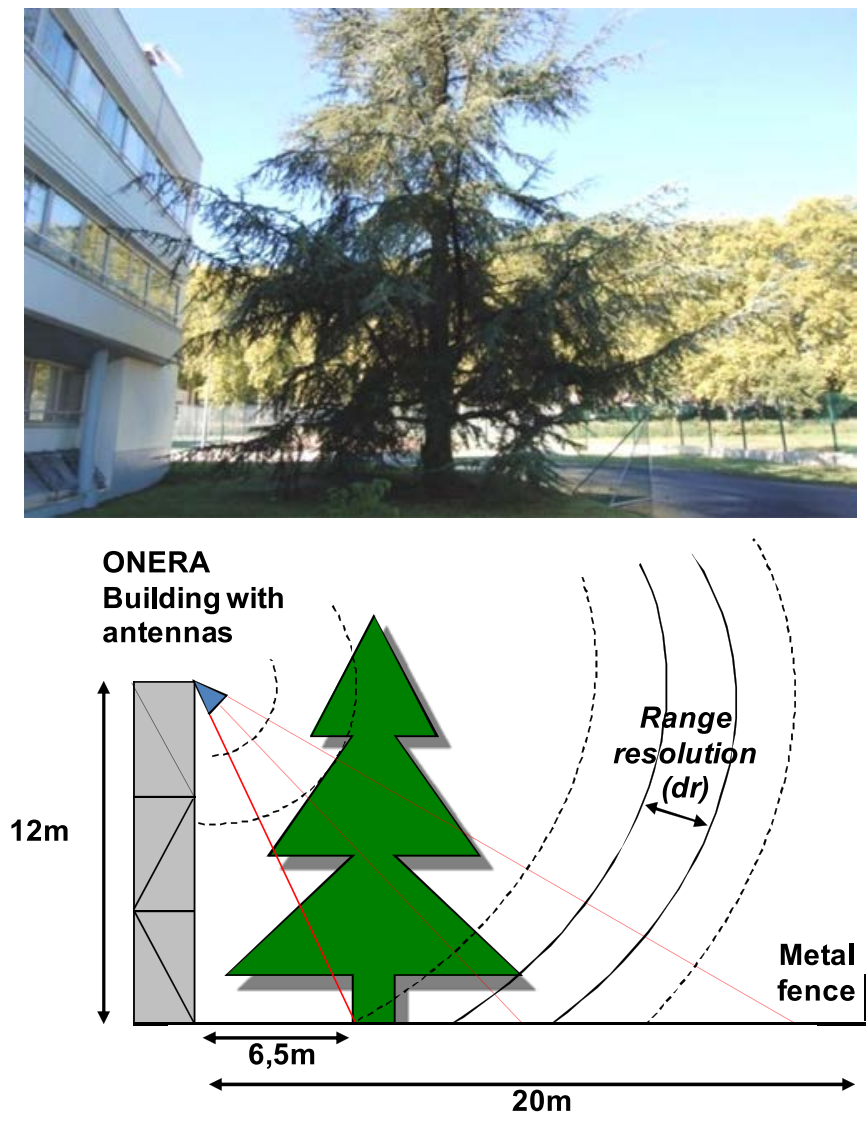

Fig. 1. (Top) Picture and (bottom) configuration of the scene.

the antenna pattern. With this experiment, it is not possible to discriminate the scatterers along the vertical direction. Therefore, the degree of reliability of the possible output data is to be determined first. This will be assessed in a feasibility study of this experiment, in this frame.

\section{A. Site Description}

The ground experiment has been installed on a building at ONERA, which makes easier an automatic acquisition of time series. This building faces a tree that can be found in temperate regions: a cedar tree (see Fig. 1). Based on the use of a vector network analyzer (VNA) and adequate antennas, the experiment may deliver P-band polarimetric radar measurements.

\section{B. Hardware}

The radar unit is based on a stepped-frequency VNA. In this experiment, the intermediate frequency bandwidth is set to $10 \mathrm{kHz}$, and the output power is set to $+3 \mathrm{dBm}$. From the hardware specifications, the dynamic range is $50 \mathrm{~dB}$.

The VNA is remotely controlled through the Ethernet network by a personal computer that is located inside the building and initiates the test, measures every $5 \mathrm{~min}$, and saves the acquired data on its hard disk.

The antennas are attached to a rail overwhelming the building wall through a gliding support, as shown in Fig. 2. They are oriented at a $60^{\circ}$ incidence angle, in both vertical and horizontal polarizations. Radio-frequency cables approximately $8 \mathrm{~m}$ long connect both antennas to the ports of the VNA. Thus, HH

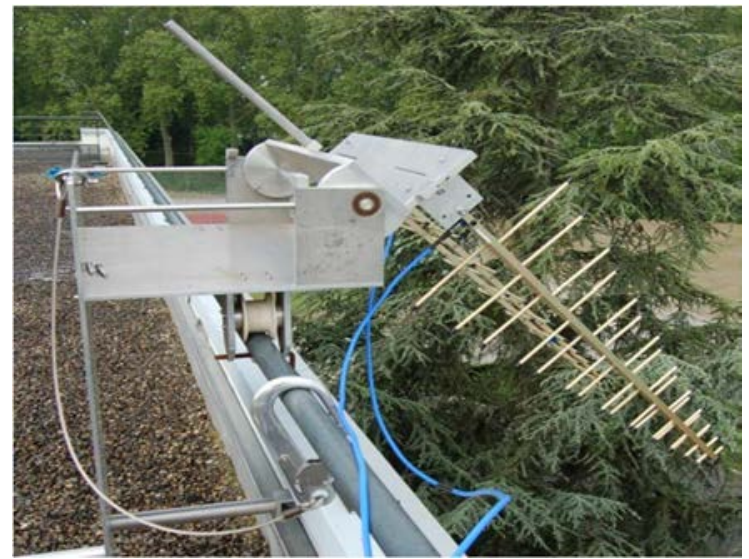

Fig. 2. Mounting of the antennas on the roof.

polarization is obtained with $\mathrm{S} 11, \mathrm{HV}$, and $\mathrm{VH}$ with $\mathrm{S} 12$ and S21, and VV with S22. The VNA measures the response for the emission of a frequency ramp between 400 and $600 \mathrm{MHz}$ and stores the results in the frequency domain.

The antennas are wide-band (from $400 \mathrm{MHz}$ to $1 \mathrm{GHz}$ ) log-periodical antennas. They are characterized by a relatively similar radiation pattern in both electric field (E-plane) and magnetizing field (H-plane), with a measured half-power beamwidth of $60^{\circ}$ for the E-plane and $80^{\circ}$ for the H-plane. In addition, the isolation between polarizations is better than $20 \mathrm{~dB}$, with very low sidelobes and backward radiation lower than $-23 \mathrm{~dB}$. Measurements of coupling between these antennas gave values lower than $-35 \mathrm{~dB}$, in the band from $400 \mathrm{MHz}$ to $1 \mathrm{GHz}$, for a spacing of $70 \mathrm{~cm}$ between two antennas. The standing wave ratio (SWR) was measured below $-13 \mathrm{~dB}$ in the band from $400 \mathrm{MHz}$ to $1 \mathrm{GHz}$ and below $-18 \mathrm{~dB}$ in the band from 400 to $600 \mathrm{MHz}$.

The main requirements for this experiment, which are tightly linked to the antenna constraints, are achieved as follows:

1) illuminating a footprint that is large enough to be statistically representative of the scene under consideration, while eliminating the spurious remote echoes;

2) eliminating as strongly as possible the echo of the metal structure of the supporting arm while illuminating the tree with the required incidence angles;

3) presenting similar radiation patterns in both E- and H-planes.

In addition, a weather station was set up and installed, with the body of the station on the roof and a soil moisture probe at the cedar base. The air temperature, the wind speed, the soil tension at $10 \mathrm{~cm}$, and the rain rate measurements were synchronized with the radar acquisitions.

\section{Acquisition Parameters}

For all the experiments presented in the sequel, the configuration parameters are the following.

1) Frequency band of measurement: $400 \mathrm{MHz} / 1 \mathrm{GHz}$. However, the results presented in this paper correspond to the P-band only $(400 / 600 \mathrm{MHz})$.

2) Full S parameters of the VNA, corresponding to Stokes matrix [8], are measured every $5 \mathrm{~min}$. 
3) Weather data are measured every $5 \mathrm{~min}$, and the rain measurements are cumulated every day so as to obtain daily rain rate.

4) Acquisition and data storage are made fully automatic.

5) A full parameter manual calibration of the VNA and a measurement of the scene, taken from the same facade but $30 \mathrm{~m}$ away so as to measure the RCS of an identical scene but without the tree, are performed every roughly ten days. The measurement of the scene without the tree is called an "empty scene."

The corner reflectors, either trihedral for $\mathrm{HH}$ and $\mathrm{VV}$ or dihedral for $\mathrm{HV}$, were located in the scene to check the correct behavior of the system.

\section{Processing}

There is no discrimination along azimuth. However, the area imaged by the radar is limited along azimuth by the antenna footprint. Range discrimination is obtained due to Fourier transform performed on the data in the frequency domain, measured on a frequency band. With $\Delta f, \delta f, N f$, which are, respectively, the frequency bandwidth, the frequency step, and the number of frequency steps $(\Delta f=(N f-1) \partial f)$, the range resolution is $d r=c / 2 \Delta f$, and the unambiguous range is $D=c / 2 \delta f$. In this experiment, as bandwidth $\Delta f$ is $200 \mathrm{MHz}$ and frequency step $d f$ is $150 \mathrm{kHz}$, then the range resolution $d r$ is $0.75 \mathrm{~m}$, and the unambiguous range $D$ is $1 \mathrm{~km}$.

Here, as only one tree is observed, the range cells do not correspond to a constant volume. However, we will not consider this particularity. For ranges intercepting the crown of the tree only, we will consider a volume clutter distribution.

To diminish the sidelobe levels, a Hamming window is applied to the measured spectrum. Thus, with $200-\mathrm{MHz}$ bandwidth, the obtained range resolution is close to $1 \mathrm{~m}$.

Then, RCS $\sigma_{0}$ is computed between ranges $r_{\min }$ and $r_{\max }$ from the complex radar response $S(t, r)$ obtained for a distance $r$ and a time $t$. To increase the number of looks, $\sigma_{0}$ was averaged over $N$ acquisitions, i.e.,

$$
\sigma_{0}(t)=\sqrt{\frac{1}{N\left(r_{\max }-r_{\min }\right)} \sum_{t=-\frac{N-1}{2}}^{\frac{N-1}{2}} \sum_{r=r_{\min }}^{r_{\max }} r|S(t, r)|^{2}} .
$$

In addition, the temporal coherence $\gamma$, which is the modulus of the complex correlation between measurements performed at two dates $t_{1}$ and $t_{2}$ and averaged on $N$ acquisitions, was also computed as in (2), shown at the bottom of the page.

When the rain pours on the antennas, the water can shortcircuit the antennas. The short-term variations of RCS, which

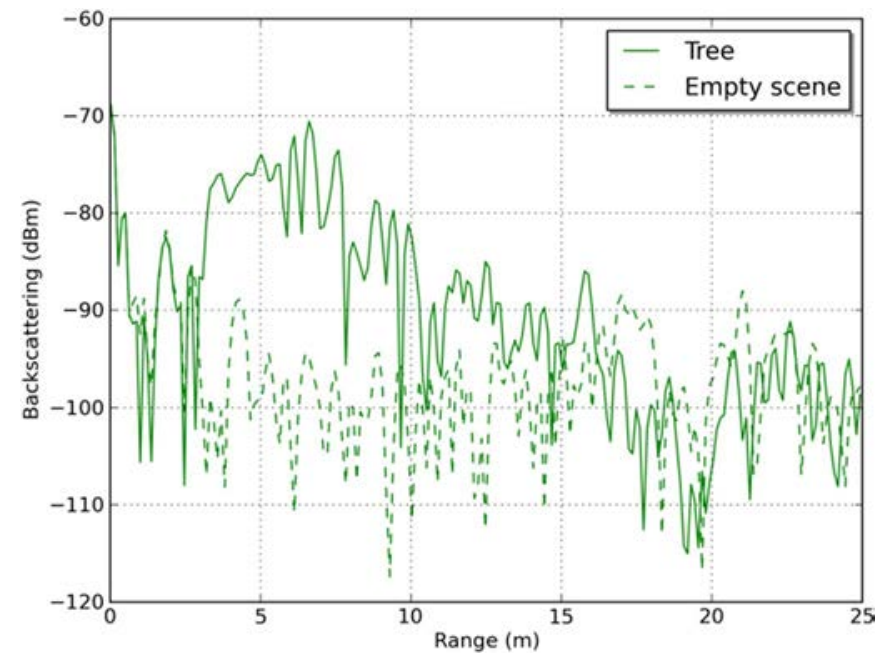

Fig. 3. Impulse response of the scene with and without the tree for polarization $\mathrm{HV}$.

appear in this case, are artificially very high, and thus, the corresponding measurements are not processed.

\section{E. Time Series}

On September 8, 2011, a short time series of $10 \mathrm{~h}$ was performed with an artificial watering of the scene and will be referred to in the following as "STS." In addition, three long time series were performed: The first time series, which is from September 9, 2011 to November 6, 2011, will be referred to as "TS-AUTUMN"; the second time series, which is from December 11, 2011 to February 19, 2012, will be referred to as "TS-WINTER"; and the third time series, which is from March 27, 2012 to June 10, 2012, will be referred to as "TS-SPRING." For the STS, 8:00 will be the reference time for the coherence. For the three long time series, midnight of the first day will be chosen as the reference date for the coherence. It has to be noted that connection problems with the network induced discontinuities during radar acquisitions of TS-AUTUMN and TS-SPRING. Moreover, the weather station was set up between TS-AUTUMN and TS-WINTER. As a consequence, rain rate and soil tension are not available for STS nor TS-AUTUMN.

\section{F. Validation of the Radar Measurements With Impulse Responses}

The system being mounted on a rail, it was translated along this rail, and some fully polarimetric measurements were performed on the same front of the building, in front of the tree, and $30 \mathrm{~m}$ away from the original position where the scene is roughly identical but without any tree.

$$
\gamma\left(t_{1}, t_{2}\right)=\frac{\sum_{t=-\frac{N-1}{2}}^{\frac{N-1}{2}} r=r_{\min }}{\sqrt{\sum_{\max }} r S\left(t_{1}, r\right) S\left(t_{2}, r\right)^{*}}
$$



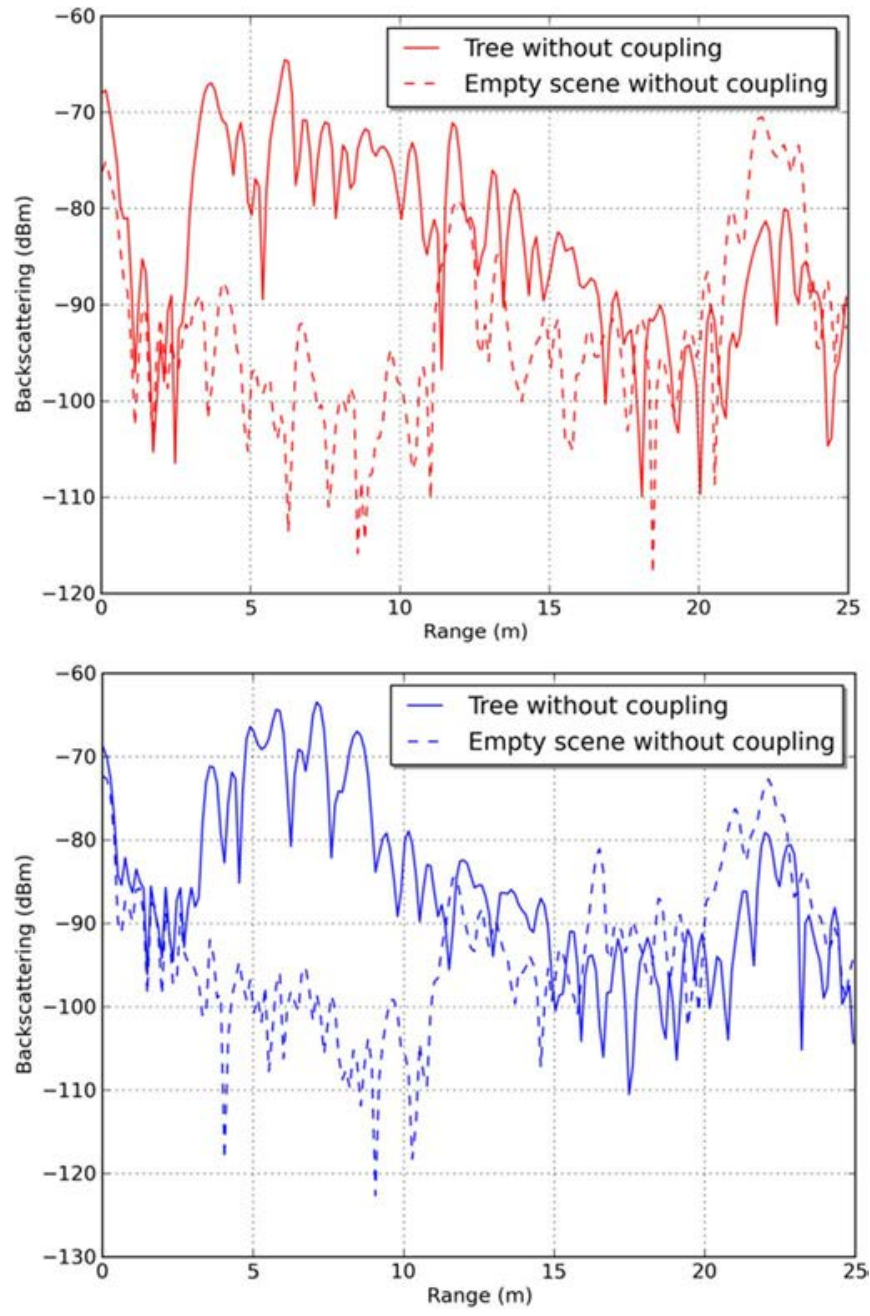

Fig. 4. Impulse response of the scene with and without the tree, with subtraction of the sky measurement, for polarizations (top) HH and (bottom) VV.

In Fig. 3, one can see that with polarization $\mathrm{HV}$, for a range between 3 and $10 \mathrm{~m}$, the tree RCS is between 10 and $20 \mathrm{~dB}$ higher than that for the empty scene. In this paper, the RCS and the coherence will be computed for the range between 3 and $10 \mathrm{~m}$, where the trunk and the branches of the tree are intercepted (the Nadir echo is located $12 \mathrm{~m}$ away from the antenna). This zone corresponds to the RCS of the canopy only.

As polarizations $\mathrm{HH}$ and VV are measured with $\mathrm{S} 11$ and S22, there are some electromagnetic reflections inside the antennas, characterized by the SWR. Hence, for polarizations $\mathrm{HH}$ and $\mathrm{VV}$, the RCS of the scene with the tree is not high enough compared with the RCS of the empty scene. To fix this issue, identical measurements were performed with the antennas facing the sky. The subtraction of the sky measurement from the scene measurement aims at correcting the coupling between antennas and the SWR of the antennas.

The corrected impulse response for polarizations $\mathrm{HH}$ and VV, as displayed in Fig. 4, shows that the tree RCS is between 10 and $20 \mathrm{~dB}$ higher than for the empty scene.

It has to be noted that the sky measurements have to be performed in a short time before or after the tree measurements, observed to be less than roughly one day, because of the temporal decorrelation introduced by the environment and the

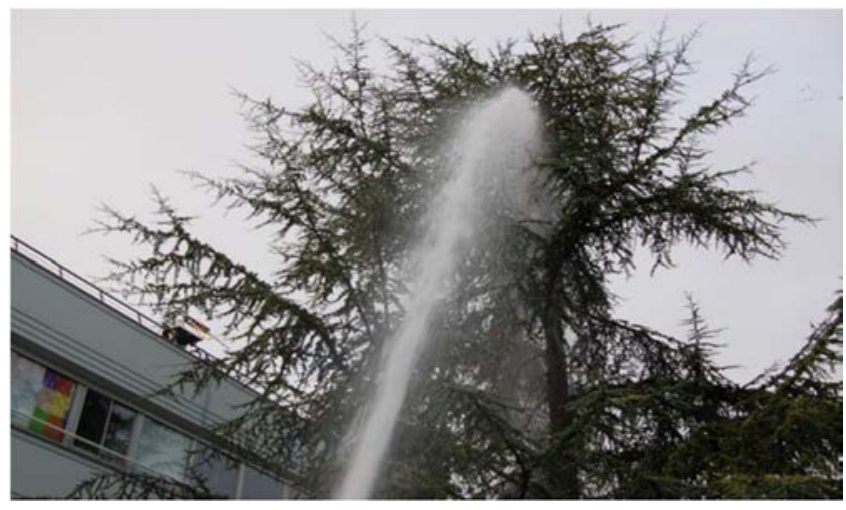

Fig. 5. Artificial watering of the tree.

system itself [9]. As a consequence, a sky measurement was performed at the beginning of the STS. This correction process results in time series exploitable for all polarizations.

For the long time series (TS-AUTUMN, TS-WINTER, and TS-SPRING), there were no sky measurements performed every day, and therefore, these time series are exploitable for polarization HV only. To obtain a good estimation of the coherence, a minimum number of independent samples is necessary: Between four and ten independent samples are needed for coherences higher than 0.8 [10]. Between 3 and $10 \mathrm{~m}$, as the spatial resolution is roughly $1 \mathrm{~m}$, the average RCS is estimated with seven independent looks. In addition, in the following of the paper, temporal averaging will be performed on $N=7$ dates, corresponding to a temporal window of $30 \mathrm{~min}$, so as to increase the number of looks to a sufficient number to properly estimate the coherence.

\section{Results FOR AN ARtificial Sensitivity Study}

During a dry period of summer, corresponding to STS, artificial watering of the scene was performed. This watering consisted in the projection of water with the help of a fire hose, as shown in Fig. 5.

The tree was watered from 8:30 A.M. to 8:40 A.M. After this time, no more watering was performed. The acquisitions have continued during the whole day, until dusk, and are displayed in Fig. 6. One can check that, as expected, watering the soil has no impact on the trunk and branch RCS. When watering the tree, the RCS is increasing for the three polarizations ( $2 \mathrm{~dB}$ for $\mathrm{HH}$ and $\mathrm{HV}$ and $3 \mathrm{~dB}$ for $\mathrm{VV}$ ) and rapidly decreasing to its previous level for $\mathrm{HV}$ and $\mathrm{VV}$, but strongly decreasing and then increasing to its previous level for $\mathrm{HH}$. The level before watering is reached again after $30 \mathrm{~min}$ for $\mathrm{HV}, 2 \mathrm{~h}$ for $\mathrm{VV}$, and $6 \mathrm{~h}$ for $\mathrm{HH}$.

Note that these watering effects may be combined with the variations in RCS with time of the day, which will be studied in the following section. This may partly explain why the drying effects are so different with polarization. Another explanation may be the architectural structure of the tree, with very large horizontal branches and thinner inclined branches. As the former is more sensitive to $\mathrm{HH}$ and the latter to $\mathrm{HV}$, it appears logical that drying effects are stronger in $\mathrm{HH}$. 

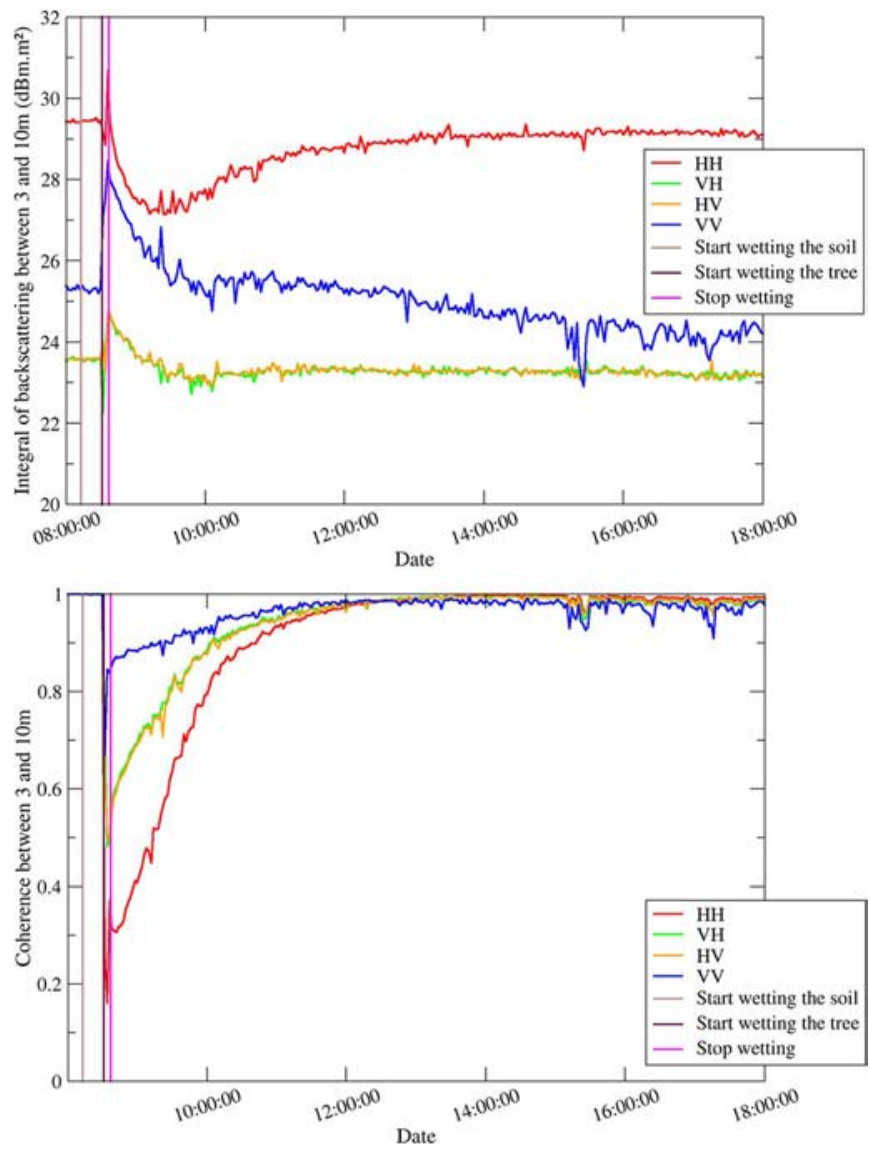

Fig. 6. Evolution of polarimetric (top) RCS and (bottom) coherence of the canopy (3-10-m range) for STS after the watering of the tree. Water was continuously project on the whole tree between the brown and pink vertical lines.

The peculiar curve of drying in $\mathrm{HH}$ is puzzling and deserves more experiments to determine if it is due to speckle effects or to a physical effect.

Concerning coherence, after maximum decorrelation during the watering of 0.7 for $\mathrm{VV}, 0.5$ for $\mathrm{HV}$, and 0.2 for $\mathrm{HH}$, a normal level of more than 0.98 is reached again for all polarizations $4 \mathrm{~h}$ after having stopped the watering. The return of the coherence to a normal value after the watering is close to an exponential curve. The analysis of this result is the same as that for the RCS.

Note that the return of polarization $\mathrm{HV}$ to its value before watering is fast for the RCS and slow for the coherence. Such behavior suggests a quick return of the dielectric values but a slow change in positions. As observed during the watering, it may be due to the branches that dry quickly and to the tufts of spines that, when they are water soaked, lower the branches because of their weight and need more time to dry. This phenomenon and the fact that $\mathrm{HH}$ is sensitive to the horizontal branches may also explain why the coherence in $\mathrm{HH}$ is the last to retrieve a high value.

\section{Results for a Natural Evolution of the Scene}

\section{A. Time Series of Autumn}

For TS-AUTUMN, we can see in Fig. 7 that diurnal cycles are visible for the coherence. Coherence remains higher than 0.8 , and at midnight every day, it presents high values, i.e.,
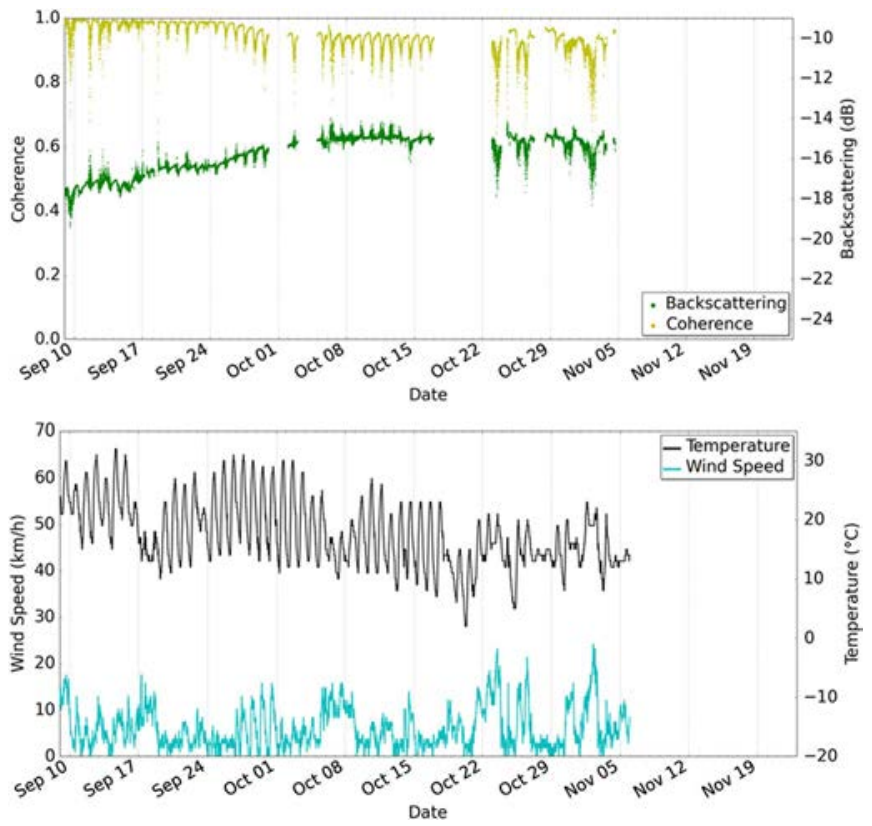

Fig. 7. (Top) RCS and coherence and (bottom) temperature and wind speed for TS-AUTUMN.

higher than 0.92. For the RCS, no cycles are visible, even if the signal is noisy during daytime. In addition, the effects of some possible rainfall can be observed on radar and weather measurement after October 24, with a strong decorrelation, similar to those previously observed during the artificial sensitivity study. For the RCS, trends are significant: There is a $1.5-\mathrm{dB}$ increase in the average RCS over the season, associated to a loss of coherence of 0.05 at its maxima. This trend seems to be correlated to the temperature: When the average temperature decreases, the average RCS increases. In addition, after a changing until early October, the average RCS value remains roughly stable.

In summary, for this period, one can observe a daily cycle for coherence only and a seasonal scale trend for both RCS and coherence.

\section{B. Time Series of Winter}

For TS-WINTER, one can see in Fig. 8 that neither the coherence nor the RCS is presenting daily cycles. The longterm trends are also more stable, with quasi-constant coherence and a monotonic increase of less than $1 \mathrm{~dB}$ over the period. This may be attributed to relatively stable weather trends: average temperature around $5{ }^{\circ} \mathrm{C}$ and rainfall distributed along time. We can observe that the rain events around December 20 induced a soil moisture content increase but have no significant impact on radar measurements. Globally, the correlation between RCS variation and rainfall is very low, which is well explained by the artificial sensitivity study.

It has to be noted that from February 2 to February 13, the air temperature is almost always between $0{ }^{\circ} \mathrm{C}$ and $-10{ }^{\circ} \mathrm{C}$, so that the branches are frozen except sometimes around 14:00. Coherence and RCS are dramatically lower and have very pronounced daily cycles: At round 14:00, RCS values close to the usual values for this period can be observed, with a dynamic 

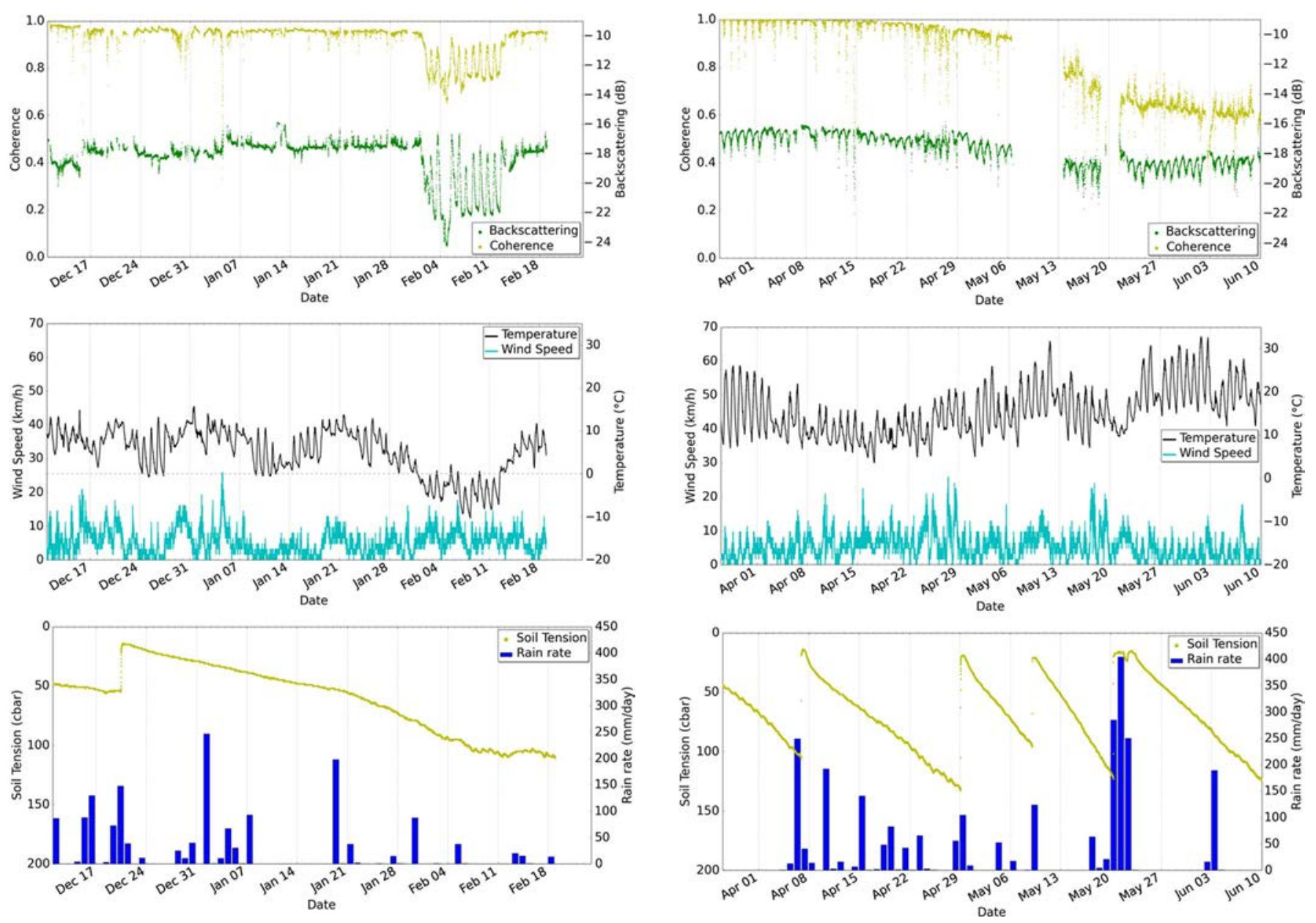

Fig. 8. (Top) RCS and coherence, (middle) temperature and wind speed, and (bottom) soil tension and rain rate for TS-WINTER.

of 4-6 dB. Such effect of frozen vegetation has already been reported on forests at the L-band with a change of $6 \mathrm{~dB}$ [11] and at the C-band with a change of $3 \mathrm{~dB}$ [12].

\section{Time Series of Spring}

For TS-SPRING, both coherence and RCS present daily cycles. These diurnal cycles on the radar measurements seem to be linked to the wind, but now, the presence of clear daily cycles on the RCS reflects a significant biophysical activity of the tree. The large-scale trend seems to be linked to the temperature. In Fig. 9, we can see on May 21 the influence of a strong rainfall that is clearly visible on the temperature and on the soil tension. These trends may be linked to changes in the evapotranspiration mechanisms. Indeed, it was previously shown that evapotranspiration mechanisms and, thus, trunk moisture content were linked to weather trends [13]. Diurnal variations of $2-3 \mathrm{~dB}$ in response to periodic fluctuations in canopy dielectric properties have been previously observed at the L-, C-, and $\mathrm{X}$-bands, during early in-situ studies of a walnut orchard growing under semiarid conditions, and airborne acquisitions performed over two half days on a loblolly pines forest showed that the time variance of the radar backscatter is found to be coupled to the measured dielectric variations [14].

Fig. 9. (Top) RCS and coherence, (middle) temperature and wind speed, and (bottom) soil tension and rain rate for TS-SPRING.

The radar measurements displayed in this paper and the differences depending on the season give us an idea of the importance of this phenomenon for a temperate cedar tree in Western Europe.

\section{Detailed Analysis}

To go further, some specific analyses have been performed for two sunny weeks without rain within the autumn and spring radar time series.

In Figs. 10 and 11, zoomed-in views of TS-AUTUMN are displayed. We can observe some very regular cycles for the coherence, with a maximal value around 9:00 and a minimal value around 17:00. This phenomenon is certainly due to the branch movements, induced by the wind peak in the afternoon, that decorrelate the signal. The RCS is rather stable even if there is more noise during the afternoon.

For zoomed-in views of TS-SPRING, as displayed in Figs. 12 and 13, we can see some very regular cycles for RCS, with a maximal value around 8:00 and a minimal value around 18:00. The coherence is rather stable during night and very noisy during daytime. Comparing with the zoomed-in views of TS-AUTUMN, it is logical to observe a lower coherence as the reference time for this coherence computation was taken earlier for TS-SPRING. 

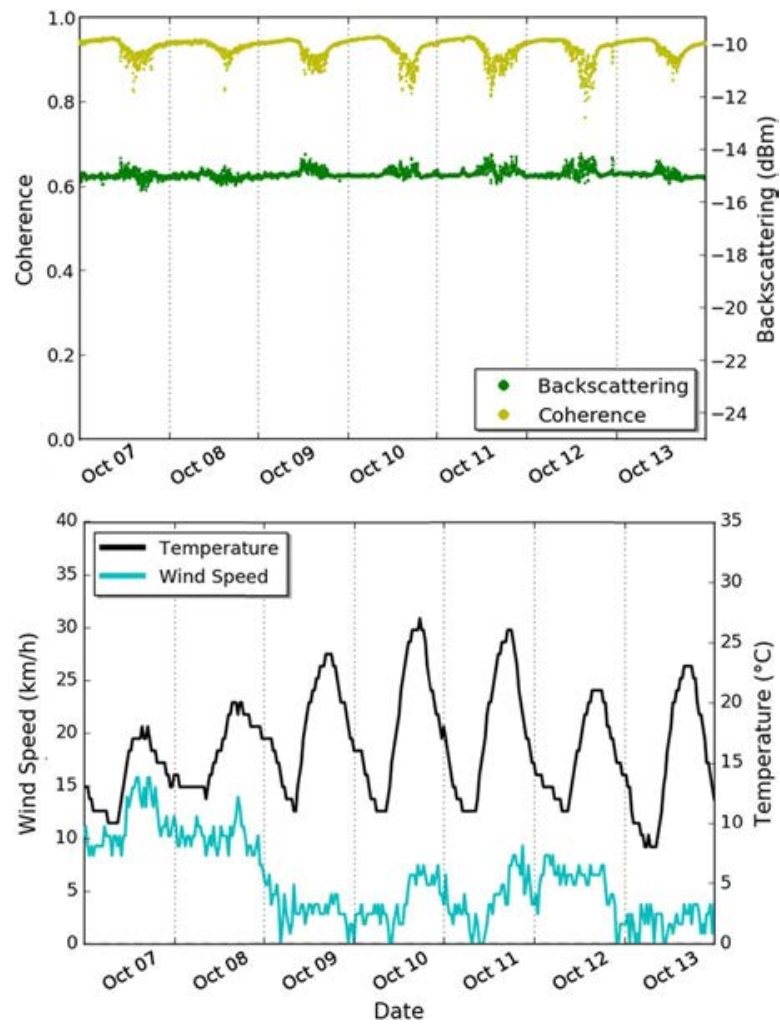

Fig. 10. Focus of (top) RCS and coherence and (bottom) temperature and wind speed, for a week without rain of TS-AUTUMN.
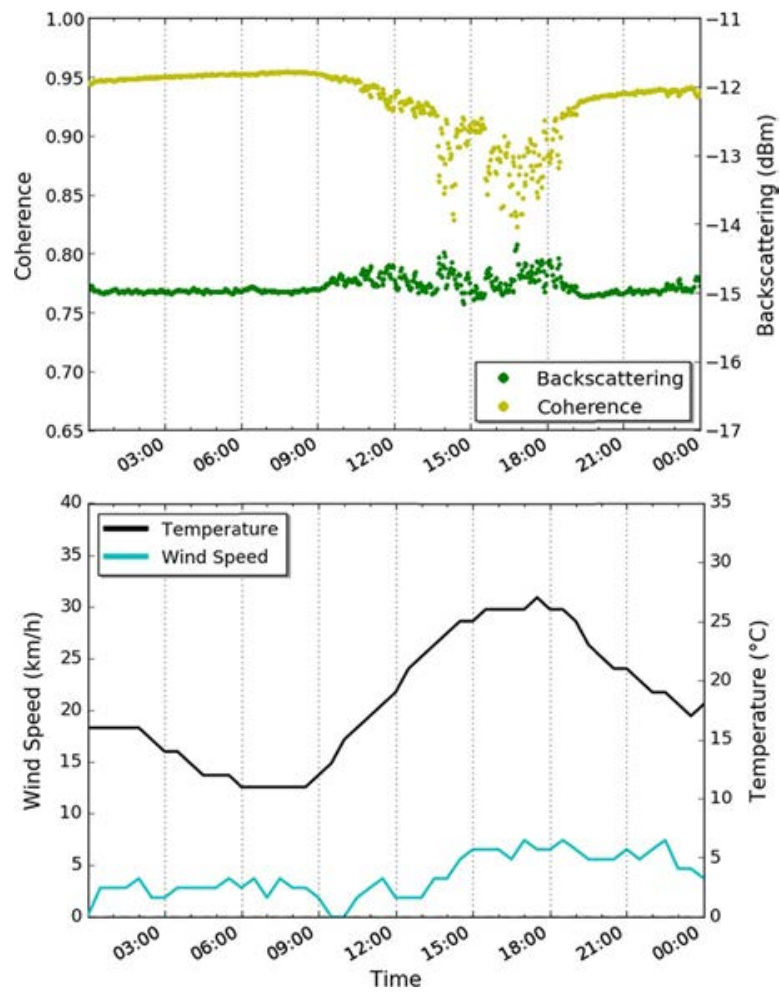

Fig. 11. Zoomed-in views of (top) RCS and coherence and (bottom) temperature and wind speed, for a day without rain of TS-AUTUMN (October 10).

However, the decorrelation events of TS-AUTUMN appear at the same time of the day as the noise in the coherence of TS-SPRING. Concerning RCS, the daily cycles in TS-SPRING that were not present for TS-AUTUMN may be due to a
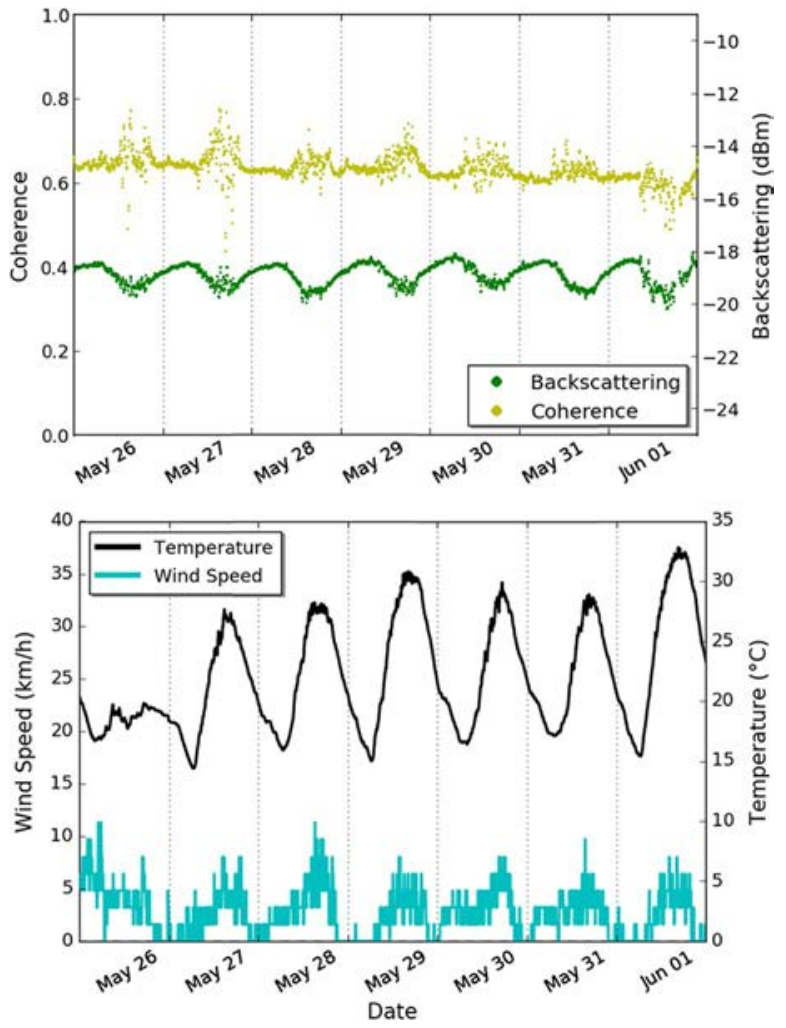

Fig. 12. Focus of (top) RCS and coherence and (bottom) temperature and wind speed, for a week without rain of TS-SPRING.
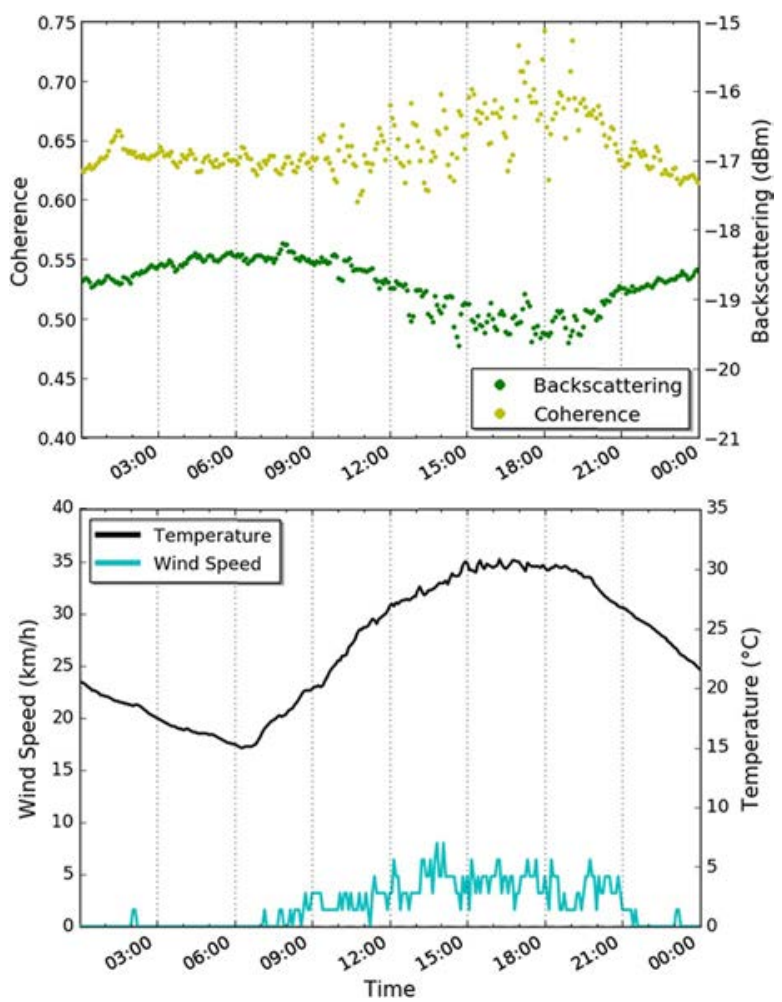

Fig. 13. Zoomed-in views of (top) RCS and coherence and (bottom) temperature and wind speed, for a day without rain of TS-SPRING (May 29).

modification in the dielectric constant daily cycles, as the two time series took place during different seasons for which tree activity is very different, i.e., October and May. 


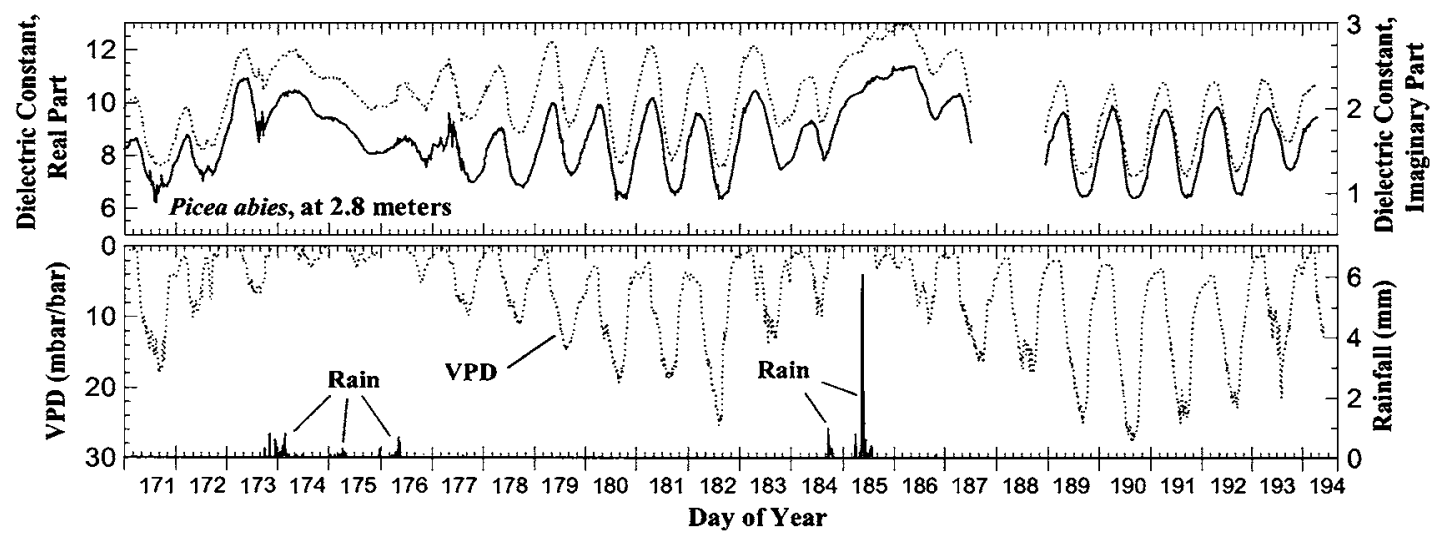

Fig. 14. Plot of $\operatorname{Re}\left(\varepsilon_{\mathrm{r}}\right)$ and $\operatorname{Im}\left(\varepsilon_{\mathrm{r}}\right)$ at the P-band for a Norway spruce (Picea Abies) at a 2.8-m height and concurrent vapor pressure deficit (VPD) and rainfall (millimeters of rainfall in 10-min intervals) plotted for time periods extending over a total of 23 days in 1995 . As the VPD and the dielectric constant tend to be negatively correlated, VPD is plotted with the zero reference placed at the top of the $y$-axis to allow for an easier comparison with dielectric variability. VPD (mbar/bar) is the measured VPD (in mbar) normalized to standard atmospheric conditions at sea level (1013 mbar)—reprint from [15].

Unfortunately, the branch dielectric constant was not measured during the experiment. To link the previous radar time series to this biophysical parameter, a reprint of a dielectric constant time series of a Norway spruce (Picea abies), in Fichtelgebirge, Germany, is displayed in Fig. 14.

For what concerns RCS, for a temperate tree, the measured dielectric constant shows diurnal cycles during dry periods, and these cycles are strongly perturbed when there is presence of rain. In addition, we can see that for daily cycles, the dielectric constant is maximum around 8:00 and minimum around 18:00, similarly to what is observed on radar measurements. Note that in [15], dielectric time series was not performed on a sufficiently long period to detect possible seasonal trends.

\section{E. Annual Changes}

With regard to seasonal changes, daily average RCS and average RCS of the tree between 6:30 A.M. and 7:30 A.M. have been computed and displayed in Fig. 15. This time corresponds to the time of acquisition of the BIOMASS mission.

During the experiment, which lasted nine months, when discarding the frozen period, the daily RCS and the RCS at 7:00 A.M. present annual changes of roughly $3 \mathrm{~dB}$ and are almost superimposed. Some similar annual changes of 2-3 dB were previously observed at the $\mathrm{C}$-band for deciduous and pine trees [16].

Variation through the year, although not fully complete, exhibits one maximum in October and another in April, to be compared with the variation in [11], which, for eastern hemlock moisture content in the trunks, presents maxima at the end of spring and in autumn.

Concerning standard deviation, the period from April to June presents higher values, which is in accordance with the previous observations of daily cycles only visible in TS-SPRING.

Note that both curves present a strong local decrease of $3 \mathrm{~dB}$ for the daily average value and of $4 \mathrm{~dB}$ for the 7:00 A.M. average value, during the period of frost. These trends may be linked to the seasonal phenomenon of the cedar, as some seasonal trends can be observed for the twig and leaf moisture
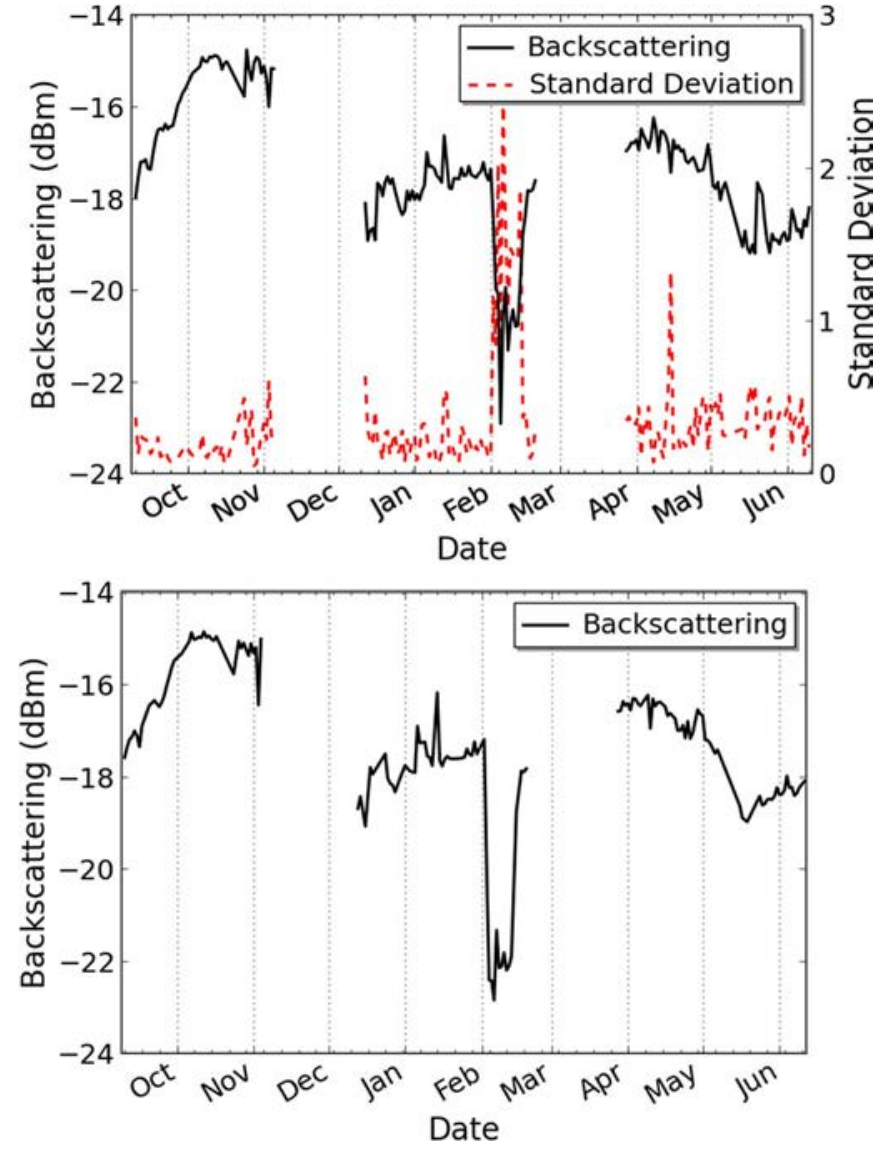

Fig. 15. (Top) Daily average RCS and (bottom) average RCS of the tree between 6:30 A.M. and 7:30 A.M., for the three periods.

content of pinyon and juniper [17] and for trunk moisture content distribution of eastern hemlock [18].

These measurements give an idea of the amplitude of annual variations of the RCS of a cedar in a temperate region.

While analyzing these results, it is important to consider the fact that only one tree was observed in a temperate region. Then, some questions appear such as the amplitude of annual 
variations of a cedar forest in temperate regions or the presence of annual variations for tropical or boreal forests.

\section{Conclusion and Future Prospects}

A system for P-band radar radiometric and coherence observation of one tree has been designed and set up. Validity tests have shown that the results for the canopy of the tree for polarization $\mathrm{HV}$ were reliable.

A first experiment with artificial watering could evidence the impact of rain on the tree response for small timescales, and it was shown that it has an effect on RCS for polarization HV that is limited in time, i.e., around $30 \mathrm{~min}$, but impacts coherence for a longer time.

During the nine months of the long-term experiment, three time series in autumn, winter, and spring have been reported and analyzed and taught us a lot. According to the season, the behavior of both RCS and coherence was found to be very different. Indeed, the coherence presents daily cycles only in autumn and in spring, whereas the RCS presents these cycles only in spring with an amplitude variation of 1-2 dB. It was stated that these cycles might be explained by cycles in the dielectric constant almost due to evapotranspiration mechanisms. In addition, the HV average RCS has a yearly cycle of roughly $3 \mathrm{~dB}$, and similar trends can be found in the time series of moisture content of trunks. The observations done here have permitted obtaining the quantitative behavior of the RCS and the coherence of a single tree at various scales of time and, thereby, a qualitative picture of the behavior of a temperate forest.

These changes have implications for retrieval biomass algorithms based on intensity or interferometric coherence [2]. The biomass algorithms based on the polarimetric RCS (PolSAR) or the multipass technics that need high coherence (PolInSAR and TomoSAR) might have to take into account the short-term changes due to rain or snow fall and the long-term variations due to the seasonal effects.

A first prospect would be to perform a similar campaign over a temperate forest. The magnitude of diurnal and seasonal changes in intensity and coherence poses some interesting challenges for SAR calibration if diurnal response is to be used to infer canopy water status. However, some reserves are to be issued on the low number of independent samples and, thus, the fact that the previous results only account for a tree and not for a forest. Future prospects should include supplementary time-series acquisitions and analyses. A deeper interpretation of the results with the help of electromagnetic modeling should be conducted. In addition, the use of a real or synthetic vertical antenna array would make it possible to discriminate the scatterers along the vertical direction and then to follow the temporal evolution of the radar backscattering along the vertical direction.

\section{ACKNOWLEDGMENT}

The authors would like to thank P. Dubois-Fernandez, from the Office National d'Etudes et de Recherches Aérospatiales, for her support to the project and all her advices.

\section{REFERENCES}

[1] "BIOMASS phase 0 report for assessment," Eur. Space Agency, Charlottesville, VA, USA, Ref. SP1313/2, Nov. 2008.

[2] T. Le Toan, A. Beaudoin, J. Riom, and D. Guyon, "Relating forest biomass to SAR data," IEEE Trans. Geosci. Remote Sens., vol. 30, no. 2, pp. 403-411, Mar. 1992.

[3] P. Borderies, L. Villard, C. Albinet, and N. Floury, "Forest microwave backscatter modeling at P Band: Temperate pine forest," in Proc. IEEE IGARSS, Jul. 22-27, 2012, pp. 3403-3406.

[4] S. R. Cloude and K. P. Papathanassiou, "Polarimetric SAR interferometry," IEEE Trans. Remote Geosci. Remote Sens., vol. 36, no. 5, pp. 1551-1565, Sep. 1998.

[5] P. Dubois-Fernandez et al., "TropiSAR: Exploring the temporal behavior of P-Band SAR data," in Proc. IEEE IGARSS, Jul. 25-30, 2010, pp. 1319-1322.

[6] C. Albinet et al., "TropiSCAT: A ground based polarimetric scatterometer experiment in tropical forests," IEEE J. Sel. Topics Appl. Earth Observ. Remote Sens., vol. 5, no. 3, pp. 1060-1066, Jun. 2012.

[7] P. Borderies, C. Albinet, and J. Besson, "Measure of temporal variation of backscattering coefficient and phase scattering by trees at P-Band," Eur. Space Agency, Charlottesville, VA, USA, Contract No. 400102822/ 10/NL/IB/fk.

[8] G. T. Ruck, D. E. Barrick, W. D. Stuart, and C. K. Krichbaum, Radar Cross Section Handbook. New York, NY, USA: Plenum, Jan. 1970

[9] H. A. Zebker and J. Villasenor, "Decorrelation in interferometric radar echoes," IEEE Trans. Geosci. Remote Sens., vol. 30, no. 5, pp. 950-959, Sep. 1992.

[10] R. Touzi, A. Lopes, J. Bruniquel, and P. W. Vachon, "Coherence estimation for SAR imagery," IEEE Trans. Geosci. Remote Sens., vol. 37, no. 1, pp. 135-149, Jan. 1999.

[11] R. Kwok, E. J. M. Rignot, J. Way, A. Freeman, and J. Holt, "Polarization signatures of frozen and thawed forests of varying environmental state," IEEE Trans. Geosci. Remote Sens., vol. 32, no. 2, pp. 371-381, Mar. 1994.

[12] E. Rignot et al., "Monitoring of environmental conditions in Taiga forests using ERS-1 SAR," Remote Sens. Environ., vol. 49, no. 2, pp. 145-154, Aug. 1994.

[13] D. M. Gates, "Water relations of forest trees," IEEE Trans. Geosci. Remote Sens., vol. 29, no. 6, pp. 836-842, Nov. 1991.

[14] M. C. Dobson, K. Mc Donald, and L. Pierce, "Diurnal variation in radar backscatter from a loblolly pine forest in late summer," in Proc. IEEE Int. Geosci. Remote Sens. Symp., 1991, pp. 1115-1116.

[15] K. C. McDonald, R. Zimmermann, and J. S. Kimball, "Diurnal and spatial variation of xylem dielectric constant in Norway spruce (Picea abies [L.] Karst.) as related to microclimate, xylem sap flow, and xylem chemistry," IEEE Trans. Geosci. Remote Sens., vol. 40, no. 9, pp. 2063-2082, Sep. 2002.

[16] C. Proisy, E. Mougin, E. Dufrêne, and V. Le Dantec, "Monitoring seasonal changes of a mixed temperate forest using ERS SAR observations," IEEE Trans. Geosci. Remote Sens., vol. 38, no. 1, pp. 540-552, Jan. 2000.

[17] D. A. Jameson, "Diurnal and seasonal fluctuations in moisture content of pinyon and juniper," Forest Service, U.S. Dept. of Agriculture, Washington, DC, USA, Rocky Mountain Forest and Range Exp. Station, 1966.

[18] J. Clark and R. Darnley Gibbs, "Further investigations of seasonal changes in moisture content of certain Canadian forest trees," Stud. Tree Physiol. IV, Can. J. Botany, vol. 35, pp. 219-253, 1957. 